\title{
GPI7 affects cell-wall protein anchorage in Saccharomyces cerevisiae and Candida albicans
}

\author{
Mathias Richard, ${ }^{1}+$ Piet de Groot, ${ }^{2}$ Olivier Courtin, ${ }^{3}$ Daniel Poulain, ${ }^{4}$ \\ Frans Klis ${ }^{2}$ and Claude Gaillardin ${ }^{1}$
}

Author for correspondence: Mathias Richard. Tel: +331308154 53. Fax: +33130815457. e-mail: richard@grignon.inra.fr

\footnotetext{
1 Laboratoire de Génétique Moléculaire et Cellulaire, Institut National Agronomique ParisGrignon, UMR-INRA216, URA-CNRS1925, BP01, 78850 Thiverval-Grignon, France

2 Fungal Research Group, Swammerdam Institute for Life Sciences, University of Amsterdam, Nieuwe Achtergracht 166, 1018 WV Amsterdam, The Netherlands

3 Aventis Pharma, 102 route de Noisy, 93235

Romainville cedex, France

4 Laboratoire de Mycologie Fondamentale et Appliquée, INSERM EPI 9915, Université de Lille II, Faculté de Médecine H. Warembourg, Pôle Recherche, Place Verdun, 59037 Lille Cedex, France
}

\begin{abstract}
Glycosylphosphatidylinositol (GPI)-anchoring represents a mechanism for attaching proteins to the cell surface of all eukaryotic cells. Two localizations of GPI proteins have been observed in the yeasts Saccharomyces cerevisiae and Candida albicans: plasma membrane and cell wall. The signals and the mechanisms involved in this differential targeting are presently not well understood. Here several cell-wall-related phenotypes of a gpi7llas21 deletion are described, where GPI7ILAS21 encodes a GPI-anchor-modifying activity. In both organisms, the structure and composition of the cell wall was modified, with a clear increase in chitin deposition. Cell-wall-targeted proteins accumulated in the growth medium, whereas the protein content of the cell wall decreased significantly, suggesting inefficiency of the covalent linkage. The level of plasma-membrane-targeted GPI proteins was not affected. Sequence analyses revealed that gene families involved in the addition of phosphoethanolamines to the core GPI anchor are highly conserved between eukaryotes, with the exception of the Gpi7 family which seems to be fungusspecific. These data are compatible with the notion that the phosphoethanolamine added by Gpi7 protein to the GPI anchor is a key factor in the covalent linkage of cell-wall proteins to fungal cell-wall components.
\end{abstract}

Keywords: glycosylphosphatidylinositol, Pir proteins, Als proteins

\section{INTRODUCTION}

Numerous glycoproteins of lower or higher eukaryotes become attached to a glycosylphosphatidylinositol (GPI) anchor, many of which ultimately appear at the plasma membrane, and in some fungi in the cell wall (Tiede et al., 1999). This anchor has the conserved core structure described in all eukaryotes: lipid- $\mathrm{PO}_{4}-m y o$-inositol$(6 \rightarrow 1)-\mathrm{GlcNH}_{2}-(4 \rightarrow 1)-\alpha \mathrm{Man}-(6 \rightarrow 1)-\alpha \mathrm{Man}-(2 \rightarrow 1)-$ $\alpha \mathrm{Man}-(6)-\mathrm{PO}_{4}-\left(\mathrm{CH}_{2}\right)_{2}-\mathrm{NH}$. In yeast, this glycan core is decorated during its assembly with a fourth mannose residue and with additional phosphoethanolamines (EtN-Ps) to form the complete precursor glycolipid (Benghezal et al., 1995; Grimme et al., 2001). The transfer of the complete precursor onto proteins occurs

†Present address: Department of Microbiology, Columbia University, 701 West 168th Street, New York, NY 10032, USA.

Abbreviations: Als, agglutinin-like sequence; ConA, Concanavalin $A$; CWP, cell-wall protein; EtN, ethanolamine; EtN-P, phosphoethanolamine; GPI, glycosylphosphatidylinositol; Pir, protein with internal repeat. in the lumen of the endoplasmic reticulum and is catalysed by a GPI transamidase complex (Ohishi et al., 2000). GPI-anchoring is not essential at a cellular level in mammalian cells, as many GPI-deficient mutant cell lines have been established (Takeda et al., 1993); however, in Saccharomyces cerevisiae GPI anchors are essential for growth (Leidich et al., 1995). Thus, all the genes known for their involvement in the biosynthesis of GPI anchors in S. cerevisiae are essential, except one, GPI7 (Benachour et al., 1999). Its deletion in S. cerevisiae hampers GPI-anchor addition to newly synthesized proteins, resulting in cell-wall fragility and mating defects (Benachour et al., 1999; Toh-e \& Oguchi, 1999). In Yarrowia lipolytica, a dimorphic yeast, the mutant cells have a clear defect in morphogenesis and display reduced invasive growth and hypersensitivity to Calcofluor White (Richard et al., 2001). Finally, in Cagpi7 mutants of Candida albicans, chlamydospore formation is affected, as well as the yeast to hypha transition on solid, but not in liquid media. The cell-wall structure is also modified in the mutants, and budding patterns, 


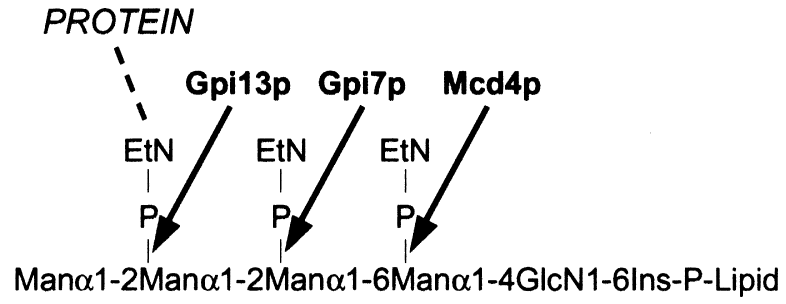

Fig. 1. Representation of the $S$. cerevisiae GPI core structure and the main proteins involved in the addition of the three EtN groups on the different mannose groups. Man, Mannose; GIcN, glucosamine; Ins, myo-inositol.

cytokinesis and cell shape are abnormal in both liquid and solid media. In vivo assays revealed that these mutants have a reduced virulence, a reduced ability to colonize the gut in mice and are more sensitive to the lytic action of macrophages (Richard et al., 2002).

S. cerevisiae is known to have a GPI core structure with a fourth mannose group; Smp3p, an essential protein, is described as being involved in the addition of this group (Grimme et al., 2001). Various studies have described three proteins involved in the transfer of EtN-Ps onto the three first mannose groups of the GPI anchor (Benachour et al., 1999; Flury et al., 2000; Taron et al., 2000). Mcd4p adds an EtN-P on the first mannose, Gpi7p on the second and Gpi13p/Yll031c on the third one (Fig. 1) (Flury et al., 2000). Whereas both MCD4 and GPI13 are essential, GPI7 is not. The lethality of the GPI13 deletion is to be expected since its gene product is involved in the addition of the EtN-P, which links the anchor to the protein. The function of the other two EtN groups is unknown. Here we present evidence that the side chain added by Gpi7p participates in the proper anchoring of GPI-modified proteins targeted to the cell wall.

\section{METHODS}

Strains and growth conditions. The C. albicans strains used were SC5314 (wild-type), MLR2A42 (ura3::imm434/ ura3::imm434, Cagpi7::hisG/Cagpi7:: hisG-CaURA3-hisG) and MLR4A2 (ura3::imm434/ura3::imm434, Cagpi7:: hisG/Cagpi7:: hisG-CaURA3-hisG) (Richard et al., 2002). The $S$. cerevisiae strains used were FY23 (MATa ura3-52, trp1D63, leu2D1) and sgpi7 (MATa, ura3-52, his3D200, LEU2, LYS2, trp1D63) (de Groot et al., 2001). Candida cells were grown at $37^{\circ} \mathrm{C}$, whereas Saccharomyces cells were grown at $30{ }^{\circ} \mathrm{C}$ in Yeast Peptone Dextrose (YPD) for preculture. During experiments, cells were cultured in Synthetic Complete (SC) medium, at different $\mathrm{pH}$ values, containing $0.17 \%$ yeast nitrogen base without amino acids (Difco), $2 \%$ glucose, $0.5 \%$ Casamino acids, $100 \mathrm{mM}$ HEPES and $0.01 \%$ uracil, histidine, tryptophan or tyrosine, and $0.02 \%$ leucine when required. Two independent transformants, MLR2A42 and MLR4A2, were included in all experiments to ensure that the phenotypic traits observed were solely due to the CaGPI7 mutation and not to unrelated mutations that may have occurred during the construction of $\Delta$ Cagpi 7 null strains. In a previous publication, we demonstrated that the phenotypes observed were due to CaGPI7 disruption (Richard et al., 2002) by reintroducing CaGPI7 in MLR2A42.

Quantazyme and chitinase sensitivity assays. These assays were carried out to detect possible changes in the organization of the 1,3- $\beta$-glucan and chitin layers of the cell wall. Quantazyme is a recombinant $1,3-\beta$-glucanase (Quantum Biotechnology). The Quantazyme sensitivity assay was conducted as described by Ovalle et al. (1998). Briefly, cells in exponential phase $\left(\mathrm{OD}_{600}=1-2\right)$ were resuspended in $40 \mathrm{mM}$ $\beta$-mercaptoethanol, $50 \mathrm{mM}$ Tris $/ \mathrm{HCl}, \mathrm{pH}=7 \cdot 5$, to an $\mathrm{OD}_{600}$ of 1 . After incubation for $1 \mathrm{~h}$, Quantazyme dissolved in glycerol/water $(1: 1, \mathrm{v} / \mathrm{v})$, was added to a final concentration of $400 \mathrm{U} \mathrm{ml}^{-1}$. Cell lysis was determined by following the decrease in turbidity at $600 \mathrm{~nm}$ over time. Quantazyme sensitivity was expressed as a percentage of the initial $\mathrm{OD}_{600}$. Streptomyces griseus chitinase (Sigma; C1525) was used in some experiments at $0 \cdot 2 \mathrm{U} \mathrm{ml}^{-1}$ for $1 \mathrm{~h}$ before Quantazyme addition.

SDS hypersensitivity tests. Exponential cultures were harvested and adjusted to an $\mathrm{OD}_{600}$ of $1 ; 5 \mu \mathrm{l}$ of tenfold serial dilutions were spotted on YPD containing increasing amount of SDS: $0 \cdot 01,0.05$ and $0 \cdot 1 \%$. Plates were read after $24 \mathrm{~h}$ culture at $30^{\circ} \mathrm{C}$ for S. cerevisiae and $37^{\circ} \mathrm{C}$ for C. albicans.

Isolation of cell-wall proteins (CWPs). For these experiments, cultures were grown in SC medium at $30^{\circ} \mathrm{C}$, pH 5.5, for $S$. cerevisiae strains, and at $37^{\circ} \mathrm{C}, \mathrm{pH} 4 \cdot 5$, for C. albicans strains, since faint differences could only be detected at higher $\mathrm{pH}$ in the CWP content of the wild-type and the gpi7 mutants of C. albicans. After $16 \mathrm{~h}$ culture in SC, cells were harvested in exponential phase $\left(\mathrm{OD}_{600}=2\right)$ and washed with $10 \mathrm{mM}$ Tris/HCl, pH 7.6, as described previously (Kapteyn et al., 1995; Montijn et al., 1994). Briefly, cell walls were isolated and washed extensively with $1 \mathrm{M} \mathrm{NaCl}$. Isolated cell walls were boiled twice in the presence of SDS, EDTA and $\beta$-mercaptoethanol to solubilize the non-covalently linked CWPs and to remove any contaminant derived from the cytosol and/or plasma membrane. SDS-extracted cell walls were freeze-dried after washing and $3 \mathrm{mg}$ of the pellets were treated either with recombinant Trichoderma harzianum endo-1,6- $\beta$-glucanase $\left(0.8 \mathrm{U} \mathrm{g}^{-1}\right)$ to release the GPI-CWPs (Kapteyn et al., 1996) or with $30 \mathrm{mM}$ ice-cold $\mathrm{NaOH}$ for $17 \mathrm{~h}$ to release the $1,6-\beta$ glucanase-resistant Pir-like-CWPs. Subsequently, 1,6- $\beta$-glucanase-treated cell walls were treated with ice-cold $30 \mathrm{mM}$ $\mathrm{NaOH}$ for $17 \mathrm{~h}$ to release the double linked CWPs. Each enzymic digestion was stopped by adding SDS at a final concentration of $0.4 \%$ and heating for $5 \mathrm{~min}$ at $100{ }^{\circ} \mathrm{C}$. Prior to heating in SDS, $\mathrm{NaOH}$-treated cell walls were first neutralized by adding acetic acid.

Analysis of CWPs. CWPs were separated by electrophoresis using linear $2 \cdot 6-20 \%$ polyacrylamide gels and transferred onto Immobilon-P polyvinylidene difluoride (PVDF, $0.45 \mu \mathrm{m}$ ) membrane (Millipore) using a wet blotting apparatus. CWPs were visualized by probing the membrane with peroxidaselabelled Concanavalin A (ConA; $0.3 \mu \mathrm{g} \mathrm{ml}^{-1}$ ) in PBS containing $3 \%$ bovine serum albumin, $2.5 \mathrm{mM} \mathrm{CaCl}_{2}$ and $2.5 \mathrm{mM}$ $\mathrm{MnCl}_{2}$ (Kapteyn et al., 2000). Western immunoblot analyses were performed with polyclonal antisera directed against 1,6$\beta$-glucan, agglutinin-like sequence (Als) proteins, Cwp1p, Pir2p, Ssr1p and Gas1p. For the antisera directed against Als proteins, Cwp1p, Pir2p, Ssr1p and Gas1p, the membranes were treated with $50 \mathrm{mM}$ periodic acid and $100 \mathrm{mM}$ sodium acetate for $30 \mathrm{~min}$ before the blocking step with $5 \%$ milk 
powder in PBS to enhance the specificity of the antisera. Binding of rabbit antibodies was assessed with goat antirabbit IgG peroxidase (Pierce) at a dilution of 1:10000 in PBS/5\% milk powder. The blots were visualized with ECL Western blotting detection reagents (Amersham Pharmacia Biotech) and Fuji Medical X-Ray film.

Determination of glucose, mannose and glucosamine content in cell walls. Isolated cell walls (about $1-1.5 \mathrm{mg}$ ) were hydrolysed as described previously with minor modifications (Dallies et al., 1998). Briefly, cell walls were hydrolysed with $50 \mu \mathrm{l} 72 \%$ sulfuric acid for $3 \mathrm{~h}$ at $25^{\circ} \mathrm{C}$. Then $650 \mu \mathrm{l}$ distilled water was added to obtain a $1 \mathrm{M} \mathrm{H}_{2} \mathrm{SO}_{4}$ solution, followed by incubation at $100{ }^{\circ} \mathrm{C}$ for $3 \mathrm{~h}$. The solution was diluted by adding $3 \mathrm{ml}$ distilled water and precipitation of sulfate was carried out using $3.3 \mathrm{ml} 40 \mathrm{~g}$ barium hydroxide $\mathrm{l}^{-1}$. After centrifugation, the sugars were quantified using a Dionex BioLC system with a CarboPac PA 1 anion-exchange column. In this experiment $5 \mathrm{mM}$ galactose was used as internal standard.

\section{RESULTS}

\section{The family of genes involved in the addition of EtN-P is highly conserved between $S$. cerevisiae and C. albicans}

To investigate whether genes involved in EtN-P addition on the GPI core structure were conserved between $S$. cerevisiae and C. albicans, an analysis of the sequence database of $C$. albicans was performed. This revealed that homologues of MCD4, GPI7 and GPI13, all forming part of a major facilitator superfamily, exist in C. albicans. They were tentatively called CaMCD4, CaGPI7 and CaGPI13, respectively. All predicted amino acid sequences exhibit significant similarities to each other both in sequence and in predicted structure. Percentages of identity vary between 22 and $38 \%$ among paralogues in a given organism and between 35 and $56 \%$ among $S$. cerevisiae and C. albicans homologues (see Fig. 2A). All predicted proteins possess between 7 and 14 predicted transmembrane domains with a large, probably luminal loop. The length and the position of the predicted loop are also conserved: its size varies between 398 and 470 aa and it begins just after the first transmembrane domain. This analysis also identified putative motifs responsible for functional specificity. The large luminal loop, which is the most conserved region among homologues (53-67.5\% identity) overlaps a predicted type I phosphodiesterase/nucleotide pyrophosphatase domain (pfam01663). Within this domain, each pair of homologues shares a very highly conserved motif of about 30 aa, which clearly permits separation of Mcd4-, Gpi7- and Gpi13-like proteins into three classes, the latter two being closely related and divergent from the first (Fig. 2B). Taken together, these results suggest that the number, structure and probable biochemical function of the enzymes involved in the addition of the three EtN-Ps onto the GPI core structure are highly conserved between $S$. cerevisiae and C. albicans.

\section{gpi7 cells exhibit marked sensitivity to SDS}

gpi7 mutants in S. cerevisiae and C. albicans have been described as being hypersensitive to Calcofluor White (Benachour et al., 1999; Richard et al., 2002), suggesting a cell-wall defect. To further support this hypothesis, we examined the sensitivity of the cells to SDS. SDS sensitivity suggests a loose structure for the cell wall, allowing SDS to reach the plasma membrane and to perturb its organization, leading to cell death. When Cagpi7 mutants were grown in the presence of increasing concentrations of SDS, they were found to be hypersensitive to SDS after $24 \mathrm{~h}$ at $37^{\circ} \mathrm{C}$ (data not shown). However, no SDS hypersensitivity was observed in the case of $S$. cerevisiae gpi7 mutants (data not shown).

\section{Composition of the mutant cell wall is modified}

To better characterize the cell-wall modification in the mutants, cell-wall preparations were hydrolysed with sulfuric acid and the released sugars were separated and quantified by high performance anion-exchange chromatography. The following results represent the mean of two independent experiments and are expressed as a percentage of the respective wild-type levels (standard
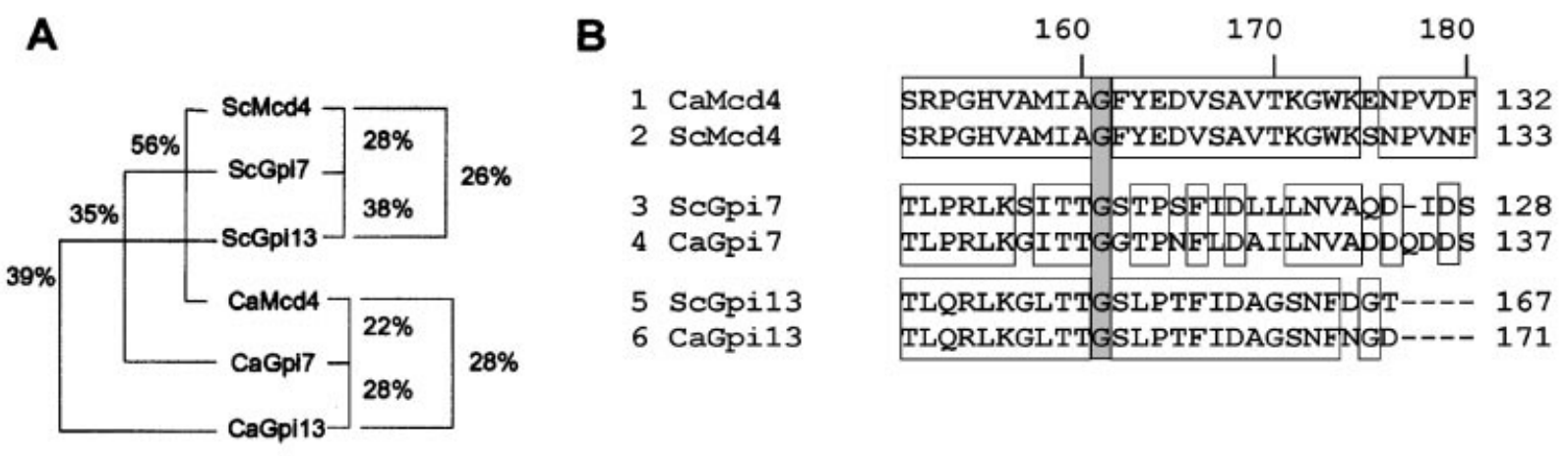

Fig. 2. Sequence comparison between $S$. cerevisiae and $C$. albicans amino acid sequences. (A) Percentage identity between MCD4, GPI7 and GPI13 homologues. (B) Alignment of the highly conserved domain in the predicted amino acids sequences of all the homologues using CLUSTAL W (Thompson et al., 1994). Identical amino acids are boxed. 




Fig. 3. Molecular architecture of the cell wall of S. cerevisiae and C. albicans. The arrows denote the orientation of the polysaccharides from the non-reducing end to the reducing end. Mature GPI-CWPs, are believed to lose their lipid moiety once they are covalently incorporated into the cell wall. GPI-CWPs can be linked by either a 1,6- $\beta$-glucan or an alkalisensitive bond, like Pir proteins, or by both. The model is based on data from Kollar et al. (1997), Kapteyn et al. (2000), Smits et al. (1999) and this study.

error on these measurements is less than $5 \%$ ). The sugar composition of the C. albicans Cagpi7 null mutant cell wall was 70, 102 and $412 \%$ for mannose, glucose and glucosamine, respectively, and 86, 116 and $261 \%$, respectively, for the $S$. cerevisiae gpi7 null mutant. The Cagpi7 null mutants showed a fourfold increase in the chitin level, suggesting compensation of a cell-wall defect by chitin deposition, as often observed in cellwall-defective mutants of S. cerevisiae (Dallies et al., 1998) and C. albicans (Kapteyn et al., 2000). Moreover, the mannose content of the cell wall decreased by $30 \%$, indicating a defect in protein mannosylation or a decrease of mannoproteins in the mutant cell wall compared to the wild-type. Similar results were observed on cell walls of a gpi7 mutant of $S$. cerevisiae, with a greater than twofold increase in chitin content, although the mannose level was marginally affected. Overall, disruption of GPI7 seems to modify the cell wall of both organisms in a qualitatively similar way, resulting in a decrease of mannoproteins, which in turn is compensated by increased chitin deposition.

We reasoned that the excess of chitin in mutant cell walls might protect cells against lytic enzymes. Indeed, we found a higher resistance of the mutant cells of $C$. albicans and S. cerevisiae to Quantazyme, a recombinant $1,3-\beta$-glucanase (data not shown). Furthermore, we checked whether a chitinase pre-treatment of the mutant may restore Quantazyme susceptibility. Although a $1 \mathrm{~h}$ treatment with chitinase alone had no effect on cell lysis under our experimental conditions, it induced a significant increase in mutant cell lysis, comparable to wildtype, during a further Quantazyme treatment in both $C$. albicans and S. cerevisiae (data not shown).

\section{Fewer proteins are linked to the cell wall in the mutant cells}

To investigate whether fewer mannoproteins were indeed present in the cell wall, as suggested by previous data on cell-wall composition, we analysed different fractions from hot SDS cell-wall extracts of C. albicans and S. cerevisiae mutant and wild-type cells grown in SC medium in the yeast form. Indeed, in both $S$. cerevisiae and C. albicans two types of CWPs have been identified that are covalently linked to $\beta$-glucan, the GPI-dependent CWPs (GPI-CWPs) and the Pir-CWPs (Fig. 3). In $S$. cerevisiae, Pir-CWPs are cross-linked to cell-wall $1,3-\beta$-glucan by a covalent link sensitive to mild alkali treatments (see Methods). GPI-CWPs are cross-linked by a 1,6- $\beta$-glucan to $1,3-\beta$-glucan (and to a lesser extent to chitin), or by an alkali-sensitive bond to $1,3-\beta$-glucan (Pir-CWPs), or by both types of bond (Kapteyn et al., 1999). A recent publication demonstrated that the general organization of the C. albicans cell wall is very similar to that of S. cerevisiae (Kapteyn et al., 2000). To characterize the effect of CaGPI7 deletion on CWPs, we compared the fractions obtained after 1,6- $\beta$-glucanase digestion, mild alkali treatment, or mild alkali treatment after 1,6- $\beta$-glucanase digestion. Lectin-blotting of wildtype $C$. albicans 1,6- $\beta$-glucanase digests, using peroxidase-linked ConA, revealed a large smear and three mannosylated protein bands with apparent molecular masses of 220, 180 and $100 \mathrm{kDa}$, respectively (Fig. 4). As expected, anti-1,6- $\beta$-glucan antiserum did not recognize any of this material, indicating that the $1,6-\beta$-glucanase did not leave any detectable 1,6- $\beta$-glucan epitope attached to the proteins (data not shown). Cagpi7 mutants exhibited a marked reduction of ConA stained material in this test, suggesting that fewer proteins were linked to the cell wall in the mutant (Fig. 4).

Hoyer and colleagues (Hoyer et al., 1995; Hoyer, 2001) have described the ALS gene family, encoding highmolecular-mass cell-surface proteins that are believed to be covalently linked to cell-wall glucans by a remnant part of the GPI anchor. Reprobing of the same samples with an anti-Als protein antiserum (Hoyer et al., 1995) revealed a band at $400 \mathrm{kDa}$ in the wild-type (Fig. 4), which was greatly reduced when mutant cells were probed. Analysis of SDS-extracted, alkali-treated cell walls with the same anti-Als protein antiserum revealed a protein band with an apparent molecular mass of $\sim 600 \mathrm{kDa}$ for both wild-type and mutant (Fig. 4), 

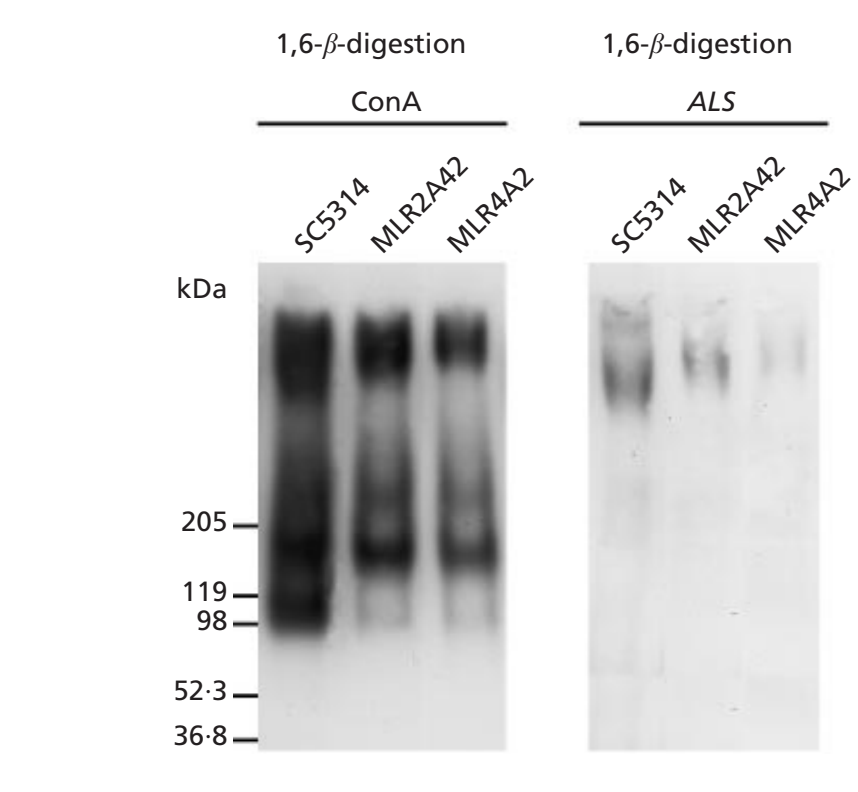

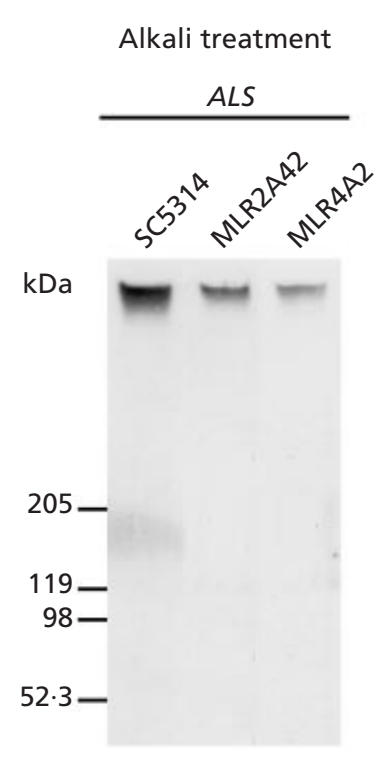

1,6- $\beta$-digestion then alkali treatment

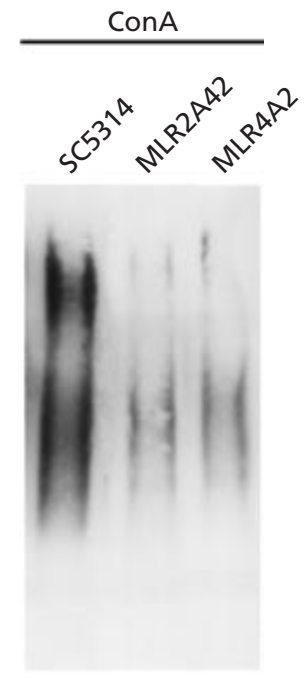

Fig. 4. Cell walls of C. albicans gpi7 contain less protein than wild-type cells. Western analysis of fractions after different treatments from wild-type (SC5314) and two independent Cagpi7 null mutants (MLR2A42 and MLR4A2) using peroxidaselabelled ConA or anti-Als protein antiserum. Each lane was loaded with the equivalent of $0.3 \mu \mathrm{g}$ dry weight of isolated SDS-extracted cell walls.

indicating that some Als proteins at least are attached by an alkali-sensitive bond. Moreover, quantitative comparison between mutant and wild-type revealed the same decrease in quantity as in the 1,6- $\beta$-glucanase digestion fraction. Finally, ConA staining of the protein fraction obtained by alkali extraction of $1,6-\beta$-glucanasedigested cell walls showed a comparable decrease in the mutant walls as seen in the other fractions (Fig. 4). Taken together, these results indicate that the cell wall of C. albicans contains GPI-dependent CWPs, including at least one Als protein, that are linked to the $1,3-\beta$ glucan network, either through 1,6- $\beta$-glucan or through an alkali-sensitive linkage, and that the cell-wall content of these proteins is markedly reduced in the Cagpi7 mutant.

\section{CWPs are released into the medium of gpi7 disrupted strains}

The above results show that mutant cell walls generally contain less GPI-CWP than the wild-type, suggesting that these proteins are either repressed, degraded or mislocalized. Preliminary results (not shown) indicate that transcription of eight $A L S$ genes tested was not modified in the mutant. We thus checked whether GPICWPs were released into the medium. Culture supernatants of the C. albicans strains were concentrated by TCA precipitation and analysed by Western blotting. Anti-Als protein and anti-Pir protein antisera revealed increased levels of putative CWPs in the culture medium of the mutant (Fig. 5a). These results indicate that these proteins are not normally linked to the cell wall in the Cagpi7 null mutant.
A similar analysis was performed for CWPs of $S$. cerevisiae, using anti-Ssr1p, anti-Cwp1p and anti-Pir $2 \mathrm{p}$ antisera. Ssr1p is a GPI-dependent CWP, which is probably associated with glucan in a structural role, and Cwp1p is a GPI cell-wall mannoprotein up-regulated by cell-wall stress. In both cases, an increased release into the growth medium of these proteins, as well as of Pir2p, was observed in the gpi7 mutant (Fig. 5b). The behaviour of Pir2p confirms previous data reporting an increase of Pir protein secretion in the case of cell-wall defects in $S$. cerevisiae (de Groot et al., 2001).

Taken together, these data demonstrate that disruption of GPI7 in C. albicans or in S. cerevisiae results in a defective cell-wall linkage of GPI-CWPs and Pir-CWPs.

\section{GPI membrane proteins are not affected by the GPI7 deletion}

We then wanted to test whether the GPI7 deletion specifically disrupted localization of GPI-CWPs, or whether it also affected proteins anchored by GPI to the cell membrane (GPI-MP). We followed the behaviour of one GPI-MP in each organism.

In $S$. cerevisiae we chose Gas1p, a GPI-modified 1,3- $\beta$ glucanosyltransferase, predominantly anchored to the plasma membrane with only traces in the cell wall (Popolo et al., 1993). SDS washes of cell-wall preparations (see Methods), known to be heavily contaminated by cell membranes (Schreuder et al., 1993), were used as a non-purified plasma membrane fraction. This crude fraction, as well as culture supernatants from $S$. cerevisiae mutant and wild-type cells, were probed with an 
(a) $A L S$

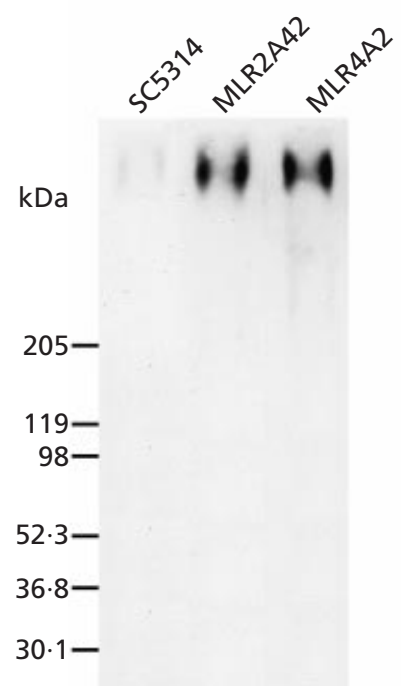

Pir-like

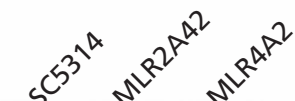

(b)
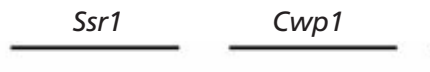

Pir2
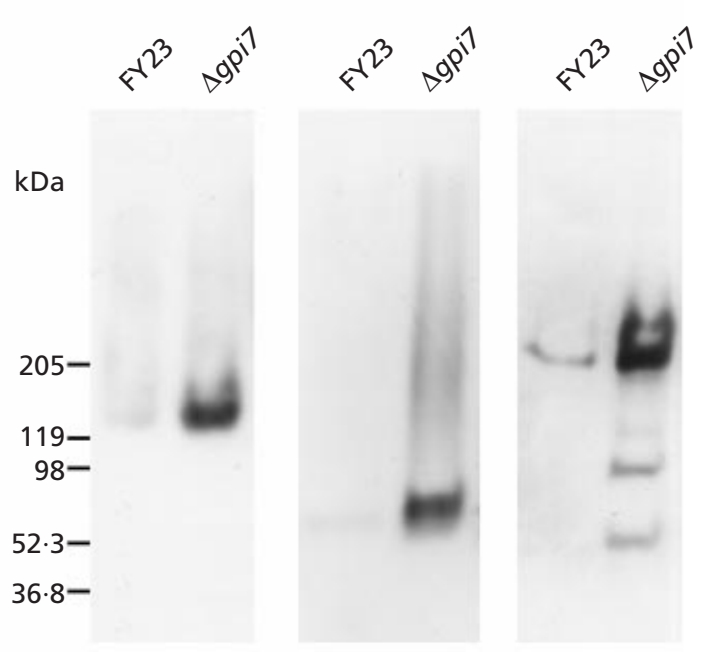

Fig. 5. Protein composition of the culture medium of wild-type and gpi7 mutants of S. cerevisiae (a) or C. albicans (b). Western analysis of protein from the culture supernatant from (a) wild-type (SC5314) and two independent Cagpi7 null mutants (MLR2A42 and MLR4A2) using peroxidase-labelled ConA, anti-Als and anti-Pir2 antisera; (b) wild-type (FY23) and gpi7 null mutant $(\Delta g p i 7)$ using anti-Ssr1p anti-Cwp1 and anti-Pir2 antisera. Each lane was loaded with proteins precipitated by TCA from $1 \mathrm{ml}$ culture medium at an $\mathrm{OD}_{600}$ of 2 .

(a) Culture
supernatant SDS extract

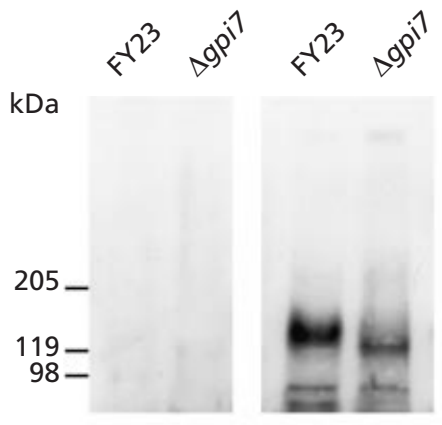

(b)

\section{Culture}

supernatant SDS extract

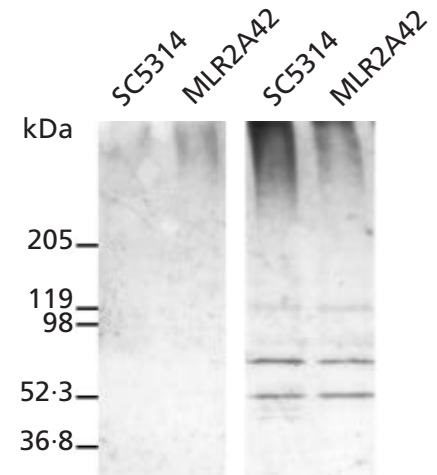

Fig. 6. GPI-MPs are not affected by the GPI7 deletion in S. cerevisiae (a) and C. albicans (b). Western analysis of protein from the culture medium from (a) wild-type (FY23) and gpi7 null mutant ( $\triangle$ gpi7) using antiGas1p antiserum; (b) wild-type (SC5314) and Cagpi7 null mutant (MLR2A42) using antiGas1p antiserum. Each lane was loaded with proteins either precipitated by TCA from $1 \mathrm{ml}$ culture medium at an $\mathrm{OD}_{600}$ of 2 or SDS-extracted from $0.2 \mu \mathrm{g}$ wet weight of cell walls.
anti-Gas1p antiserum (Fig. 6a). No signal was detected in the culture supernatants from wild-type and mutant strains. In contrast, a protein band was present in the membrane fraction of both strains at the expected size of $125 \mathrm{kDa}$ and in nearly identical amounts. These data suggest normal anchorage of at least one GPI-MP to the cell surface in the $S$. cerevisiae gpi7 mutant.

A previous study (Popolo \& Vai, 1998) demonstrated that anti-Gas1p antiserum cross-reacted with the homologues of Gas1p in C. albicans, the Phr1/2/3 proteins. To extend the results obtained in $S$. cerevisiae to $C$. albicans, an SDS extract of C. albicans cell walls was analysed using the same anti-Gas1p antiserum (Fig. 6b).
In both wild-type and mutant similar patterns were obtained: no signal was detected in the culture supernatants, whereas in the SDS extract two clear protein bands and a third, fainter band were detected at the expected positions for $\mathrm{Phr}$ proteins. These results suggest that in both organisms membrane anchoring of at least Gas1p and its homologues, and probably other GPI-MPs, is not affected by the deletion of GPI7.

\section{DISCUSSION}

In this study we investigated the potential role of the EtN-P moiety added by Gpi7p in GPI-anchoring in two 
(A)

1 scMcd4
2 CaMcd4
3 S. pombe
4 M. musculus
5 H. sapiens
6 A. thaliana
7 D. melanogaster
Consensus
1 ScGpil3
2 CaGpil13
3 M. musculus
4 H. sapiens
5 S. pombe
6 D. melanogaster
7 C. elegans
Consensus

(B)

1 ScGpi7

2 SbGpi7

3 CaGpi7

4 Y1Gpi 7

s. pombe

Consensus

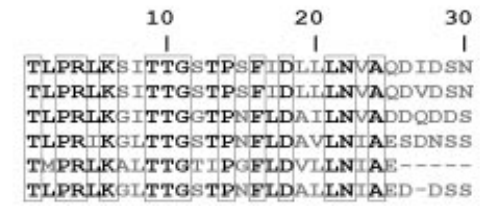

Fig. 7. Alignment of the highly conserved domain in the predicted amino acid sequences of MCD4 and GPI3 (A) and GPI7 (B) with homologues from the non-redundant sequence database.

different organisms, S. cerevisiae and C. albicans. In a first step, we scanned the $C$. albicans sequence database and found homologues for the three genes, MCD4, GPI7 and GPI13, involved in the addition of EtN-P to the GPI core structure. Interestingly, the $C$. albicans database contains a single homologue for each of these three genes. Although we confirmed the conservation of the biochemical function only for CaGpi7p (Richard et al., 2002), we speculate that the function of CaMcd4p and CaGpi13p is also conserved in view of their strong similarity to their homologues in $S$. cerevisiae. Within the conserved phosphodiesterase/nucleotide pyrophosphatase domain shared by all these proteins, a motif of about 30 aa is highly conserved between each of the three pairs of homologues and permits a clear discrimination of the three paralogues. An attractive hypothesis is that these motifs may determine the binding specificity of the proteins for each of the three mannose groups of the GPI core structure: Mcd4p on the first, Gpi7p on the second and Gpi13p on the third. Several potential homologues of the three genes can be found in lower and higher eukaryotes. As shown in Fig. $7(\mathrm{~A})$, the 30 aa motif is well conserved in most of them, permitting identification of four excellent matches with proteins from Schizosaccharomyces pombe, Drosophila melanogaster, mice and humans for both the Mcd4p and Gpi13p motifs, from Arabidopsis thaliana for the Mcd4p motif and from Caenorhabditis elegans for the Gpi13p motif. Interestingly, one EtN-P is added on the third mannose of GPI anchors in all eukaryotes and in most cases on the first one, paralleling conservation of Gpi13p and Mcd4p. On the other hand, the modification of the second mannose carried out by Gpi7p in yeast is more variable. We have noticed that Gpi7p tends to be more divergent. Closely related relatives are found only among proteins of fungal origin, from Yarrowia lipolytica, Saccharomyces bayanus and Schizosaccharomyces pombe (Fig. 7B). More distant ones are detected among humans, mice, Drosophila and Caenorhabditis proteins, where the Gpi7p motif is interrupted by two insertions of 9 and 5 aa (not shown). This suggests that Gpi7 proteins have a conserved specific function in fungi, whereas their homologues in higher eukaryotes carry out different GPI-anchor modifications.

It has been demonstrated that many GPI proteins of yeast are CWPs or plasma-membrane proteins participating in the building of the cell wall (Caro et al., 1997; Hamada et al., 1998). The phenotype of GPI7-depleted cells strongly suggests that Gpi7 affects cell-wall structure. Increased cell-wall fragility, a common feature of all gpi mutants, already suggested by previous tests with Calcofluor White (Richard et al., 2002), was confirmed by SDS hypersensitivity (Benghezal et al., 1995; Vossen et al., 1997) and we observed direct evidence of a difference in chitin content in the cell wall of mutant $C$. albicans and $S$. cerevisiae cells.

This defect in cell-wall structure correlates with a lower protein content of the cell wall in C. albicans and an increased release of CWPs in the growth medium of the mutant. Moreover, mutations in GPI7 do not affect localization of the cell membrane-attached GPI protein Gas1p.

The lower levels of GPI-dependent CWPs can be explained in different ways. First, the lack of EtN-P on the second mannose of the GPI core structure might affect the transport of GPI-dependent CWPs through the secretory pathway. This does not seem to be the case since the growth rate and the quantity of Gas1p in the plasma membrane were not affected. Second, the lack of EtN-P might block the release of the plasma-membranebound intermediate form of GPI proteins destined for the cell wall. This seems unlikely because in the mutant more soluble forms were found in the culture medium. Third, this side-chain might be essential for efficient linkage of GPI-CWPs to other cell-wall components. Thus, the lack of this side-chain may trigger mislocalization of GPI-CWPs, including some proteins involved in cell-wall synthesis and in the linkage of Pir proteins. Consequently, a defective cell wall is formed, the cell aggregates, morphogenesis is affected and virulence and the resistance to macrophages are also affected (Richard et al., 2002).

Finally, although gpi7 mutants of $S$. cerevisiae and $C$. albicans share common phenotypes, like secretion of GPI-CWPs, resistance to Quantazyme and increased chitin deposition, they also differ. In particular, no SDS hypersensitivity was detected in $S$. cerevisiae mutants and the amount of proteins in the cell wall was negligibly affected. Possibly, these phenotypic differences are due to the different growth temperatures used for $S$. cerevisiae $\left(30^{\circ} \mathrm{C}\right)$ and $\mathrm{C}$. albicans $\left(37^{\circ} \mathrm{C}\right)$. 


\section{ACKNOWLEDGEMENTS}

We thank A. Derniaux for making the cell-wall extraction and the cell-wall sugars quantification. We also thank A. Lépingle for her work on the Candida membranes. We are grateful to L. Hoyer for providing anti-Als antisera. This work was supported by the French Ministère de la Recherche (Réseau Infections Fongiques, PRFMMIP), the European Commission (QLRT-1999-30795), an EC grant to P.G. and a CNRSAventis Pharma grant to M.R.

\section{REFERENCES}

Benachour, A., Sipos, G., Flury, I., Reggiori, F., Canivenc-Gansel, E., Vionnet, C., Conzelmann, A. \& Benghezal, M. (1999). Deletion of GPI7, a yeast gene required for addition of a side chain to the glycosylphosphatidylinositol (GPI) core structure, affects GPI protein transport, remodeling, and cell wall integrity. J Biol Chem 274, 15251-15261.

Benghezal, M., Lipke, P. N. \& Conzelmann, A. (1995). Identification of six complementation classes involved in the biosynthesis of glycosylphosphatidylinositol anchors in Saccharomyces cerevisiae. J Cell Biol 130, 1333-1344.

Caro, L. H., Tettelin, H., Vossen, J. H., Ram, A. F., van den Ende, H. \& Klis, F. M. (1997). In silico identification of glycosyl-phosphatidylinositol-anchored plasma-membrane and cell wall proteins of Saccharomyces cerevisiae. Yeast 13, 1477-1489.

Dallies, N., Francois, J. \& Paquet, v. (1998). A new method for quantitative determination of polysaccharides in the yeast cell wall. Application to the cell wall defective mutants of Saccharomyces cerevisiae. Yeast 14, 1297-1306.

de Groot, P., Ruiz, C., Vazquez de Aldana, C. R. \& 13 other authors (2001). A genomic approach for identification and classification of genes involved in the cell wall formation and its regulation in Saccharomyces cerevisiae. Comp Funct Genomics 2, 1-19.

Flury, I., Benachour, A. \& Conzelmann, A. (2000). YLL031c belongs to a novel family of membrane proteins involved in the transfer of ethanolaminephosphate onto the core structure of glycosylphosphatidylinositol anchors in yeast. J Biol Chem 275, 24458-24465.

Grimme, S. J., Westfall, B. A., Wiedman, J. M., Taron, C. H. \& Orlean, P. (2001). The essential smp3 protein is required for addition of the side-branching fourth mannose during assembly of yeast glycosylphosphatidylinositols. J Biol Chem 276, 2773127739.

Hamada, K., Fukuchi, S., Arisawa, M., Baba, M. \& Kitada, K. (1998). Screening for glycosylphosphatidylinositol (GPI)-dependent cell wall proteins in Saccharomyces cerevisiae. Mol Gen Genet 258, 53-59.

Hoyer, L. L. (2001). The ALS gene family of Candida albicans. Trends Microbiol 9, 176-180.

Hoyer, L. L., Scherer, S., Shatzman, A. R. \& Livi, G. P. (1995). Candida albicans ALS1: domains related to a Saccharomyces cerevisiae sexual agglutinin separated by a repeating motif. Mol Microbiol 15, 39-54.

Kapteyn, J. C., Montijn, R. C., Dijkgraaf, G. J., Van den Ende, H. \& Klis, F. M. (1995). Covalent association of beta-1,3-glucan with beta-1,6-glucosylated mannoproteins in cell walls of Candida albicans. J Bacteriol 177, 3788-3792.

Kapteyn, J. C., Montijn, R. C., Vink, E., de la Cruz, J., Llobell, A., Douwes, J. E., Shimoi, H., Lipke, P. N. \& Klis, F. M. (1996). Retention of Saccharomyces cerevisiae cell wall proteins through a phosphodiester-linked beta-1,3-/beta-1,6-glucan heteropolymer. Glycobiology 6, 337-345.

Kapteyn, J. C., van den Ende, H. \& Klis, F. M. (1999). The contribution of cell wall proteins to the organization of the yeast cell wall. Biochim Biophys Acta 1426, 373-383.

Kapteyn, J. C., Hoyer, L. L., Hecht, J. E., Muller, W. H., Andel, A., Verkleij, A. J., Makarow, M., Van Den Ende, H. \& Klis, F. M. (2000). The cell wall architecture of Candida albicans wild-type cells and cell wall-defective mutants. Mol Microbiol 35, 601-611.

Kollar, R., Reinhold, B. B., Petrakova, E., Yeh, H. J., Ashwell, G., Drgonova, J., Kapteyn, J. C., Klis, F. M. \& Cabib, E. (1997). Architecture of the yeast cell wall. Beta $(1 \rightarrow 6)$-glucan interconnects mannoprotein, beta $(1 \rightarrow 3)$-glucan, and chitin. J Biol Chem 272, 17762-17775.

Leidich, S. D., Kostova, Z., Latek, R. R., Costello, L. C., Drapp, D. A., Gray, W., Fassler, J. S. \& Orlean, P. (1995). Temperaturesensitive yeast Gpi anchoring mutants gpi2 and gpi3 are defective in the synthesis of $\mathrm{N}$-acetylglucosaminyl phosphatidylinositol. Cloning of the GPI2 gene. J Biol Chem 270, 13029-13035.

Montijn, R. C., van Rinsum, J., van Schagen, F. A. \& Klis, F. M. (1994). Glucomannoproteins in the cell wall of Saccharomyces cerevisiae contain a novel type of carbohydrate side chain. J Biol Chem 269, 19338-19342.

Ohishi, K., Inoue, N., Maeda, Y., Takeda, J., Riezman, H. \& Kinoshita, T. (2000). Gaa1p and Gpi8p are components of a glycosylphosphatidylinositol (GPI) transamidase that mediates attachment of Gpi to proteins. Mol Biol Cell 11, 1523-1533.

Ovalle, R., Lim, S. T., Holder, B., Jue, C. K., Moore, C. W. \& Lipke, P. N. (1998). A spheroplast rate assay for determination of cell wall integrity in yeast. Yeast 14, 1159-1166.

Popolo, L. \& Vai, M. (1998). Defects in assembly of the extracellular matrix are responsible for altered morphogenesis of a Candida albicans phr1 mutant. J Bacteriol 180, 163-166.

Popolo, L., Vai, M., Gatti, E., Porello, S., Bonfante, P., Balestrini, R. \& Alberghina, L. (1993). Physiological analysis of mutants indicates involvement of the Saccharomyces cerevisiae GPIanchored protein gp115 in morphogenesis and cell separation. $J$ Bacteriol 175, 1879-1885.

Richard, M., Rosas-Quijano, R., Bezzate, S., Bordon-Pallier, F. \& Gaillardin, C. (2001). Tagging morphogenetic genes by insertional mutagenesis in the yeast Yarrowia lipolytica. J Bacteriol 183, 3098-3107.

Richard, M., Ibata Ombetta, S., Dromer, F., Bordon-Pallier, F., Jouault, T. \& Gaillardin, C. (2002). Complete glycosylphosphatidylinositol anchors are required for full morphogenesis and virulence in Candida albicans. Mol Microbiol 44, 841-853.

Schreuder, M. P., Brekelmans, S., van den Ende, H. \& Klis, F. M. (1993). Targeting of a heterologous protein to the cell wall of Saccharomyces cerevisiae. Yeast 9, 399-409.

Smits, G. J., Kapteyn, J. C., van den Ende, H. \& Klis, F. M. (1999). Cell wall dynamics in yeast. Curr Opin Microbiol 2, 348-352.

Takeda, J., Miyata, T., Kawagoe, K., lida, Y., Endo, Y., Fujita, T., Takahashi, M., Kitani, T. \& Kinoshita, T. (1993). Deficiency of the Gpi anchor caused by a somatic mutation of the PIG-A gene in paroxysmal nocturnal hemoglobinuria. Cell 73, 703-711.

Taron, C. H., Wiedman, J. M., Grimme, S. J. \& Orlean, P. (2000). Glycosylphosphatidylinositol biosynthesis defects in Gpi11p- and Gpi13p-deficient yeast suggest a branched pathway and implicate Gpi13p in phosphoethanolamine transfer to the third mannose. Mol Biol Cell 11, 1611-1630.

Thompson, J. D., Higgins, D. G. \& Gibson, T. J. (1994). CLUSTAL w : improving the sensitivity of progressive multiple sequence align- 
ment through sequence weighting, position-specific gap penalties and weight matrix choice. Nucleic Acids Res 22, 4673-4680.

Tiede, A., Bastisch, I., Schubert, J., Orlean, P. \& Schmidt, R. E. (1999). Biosynthesis of glycosylphosphatidylinositols in mammals and unicellular microbes. Biol Chem 380, 503-523.

Toh-e, A. \& Oguchi, T. (1999). Las21 participates in extracellular/cell surface phenomena in Saccharomyces cerevisiae. Genes Genet Syst 74, 241-256.
Vossen, J. H., Muller, W. H., Lipke, P. N. \& Klis, F. M. (1997). Restrictive glycosylphosphatidylinositol anchor synthesis in cwh6/gpi3 yeast cells causes aberrant biogenesis of cell wall proteins. J Bacteriol 179, 2202-2209.

Received 4 December 2001; revised 6 February 2002; accepted 4 March 2002. 